\title{
Endovascular Therapy for the Management of Acute Ilio-femoral Deep Vein Thrombosis
}

\section{Endovaskuläre Therapie zur Behandlung der akuten iliofemoralen tiefen Venenthrombose}

Author

Mert Dumantepe

Affiliation

Uskudar University School of Medicine, Department of Cardiovascular Surgery, Istanbul, Turkey

Key words

post-thrombotic syndrome, deep vein thrombosis, catheter-directed thrombolysis, endovascular therapy, pharmaco-mechanic thrombolysis, mechanic thrombectomy

Schlüsselwörter

postthrombotisches Syndrom, tiefe Venenthrombose, Katheter-gesteuerte Thrombolyse, endovaskuläre Therapie, pharmakomechanische Thrombolyse, mechanische Thrombektomie

published online 05.07 .2021

Bibliography

Phlebologie 2021; 50: 270-278

DOI 10.1055/a-1519-9344

ISSN 0939-978X

(C) 2021. Thieme. All rights reserved.

Georg Thieme Verlag KG, Rüdigerstraße 14,

70469 Stuttgart, Germany

\section{Correspondence}

Mert Dumantepe

Barbaros Mah. Gül Sokak, Agaoglu Starland Sitesi, E-2 Blok, D:43, Batı Ataşehir, Istanbul, Turkey

mdumantepe@gmail.com

\section{ZUSAMMENFASSUNG}

Bei der iliofemoralen tiefen Venenthrombose (TVT) besteht eine hohe Langzeit-Morbiditätsrate in Form des postthrombotischen Syndroms (PTS). Daher sollte sich die Behandlung einer akuten Thrombose nicht nur auf die Verhinderung von akuten Komplikationen wie Verschleppung oder Embolisation des ursprünglichen Gerinnsels konzentrieren, sondern auch auf die Verhinderung von rezidivierenden Thrombosen und PTS. Die aktuellen Katheter-gestützten Behandlungen der tiefen Venenthrombose haben sich bei bestimmten Patienten als sicher und wirksam erwiesen. Die aktuellen Leitlinien empfehlen eine medikamentöse Therapie mit Antikoagulation allein für alle außer der schwersten, die Gliedmaßen bedrohenden Thrombose. Sie ermöglichen zusätzlich die Einbeziehung einer endovaskulären Katheter-gestützten Behandlung bei bestimmten Patienten mit akuter proximaler iliofemoraler TVT und geringem Risiko von Blutungskomplikationen zur Verhinderung eines PTS.

Eine bildgebungsgesteuerte, Katheter-gestützte endovaskuläre Therapie wird bei bestimmten Patienten eingesetzt, um diese Folgeerscheinungen zu lindern, aber es bestehen nach wie vor wichtige Fragen zu ihrem optimalen Einsatz. In diesem Artikel werden die vorliegenden Erkenntnisse betrachtet und die Gründe für den Einsatz der Katheter-gestützten Therapie bei speziellen Patientengruppen mit akuter iliofemoraler TVT zusammengefasst.

\section{ABSTRACT}

Ilio-femoral deep vein thrombosis (DVT) has a high rate of long-term morbidity in the form of the postthrombotic syndrome (PTS). Therefore, management of acute thrombosis should not only focus on the prevention of acute complications such as propagation or embolisation of the initial clot but also on preventing recurrent thrombosis and PTS. Contemporary catheter-based treatments of deep vein thrombosis have proven to be safe and effective in selected patients. Current guidelines recommend medical therapy with anticoagulation alone for all but the most severe, limb-threatening thrombosis. They additionally allow for consideration of endovascular catheter-based treatment in selected patients with acute proximal ilio-femoral DVT and low risk of bleeding complications to prevent PTS.

Imaging-guided, catheter-based endovascular therapy has been used in selected patients to alleviate these sequelae, but important questions remain about their optimal use. In this article, we review the available evidence and summarize the rationale for use of catheter-based therapy in specific patient groups with acute iliofemoral DVT. 


\section{Introduction}

An acute deep vein thrombosis (DVT) refers to an episode of venous thrombosis for which symptoms have been present for 14 days or less or for which imaging studies confirm that the venous thrombosis occurred within the last 14 days [1]. With an estimated population incidence of 1:1000 persons each year, acute DVT of the lower extremities is associated with significant morbidity and a high rate of recurrence [2, 3].

Frequently presenting with non-specific symptoms such as pain, swelling, and tenderness of the affected extremity, the early course of DVT can vary among patients, ranging from complete resolution, development of severe complications such as acute limb threat, renal vein thrombosis, or even death by pulmonary embolism (PE) [4, 5]. Anticoagulant therapy is usually effective in preventing these complications; however, even with optimal initial management, there remains a substantial risk of developing post-thrombotic syndrome (PTS), including or not including venous ulcers, as a long-term complication $[6,7]$.

The conventional conservative treatment of acute DVT aims to prevent thrombus propagation and to reduce the initial symptoms and the risk of PE and DVT recurrence [8]. However, this treatment is ineffective at reducing thrombus burden in patients with proximal ilio-femoral DVT and consequently does not sufficiently prevent PTS [9]. Natural history studies of treatment with anticoagulation alone for ilio-femoral DVT have shown that, at 5 years, over $90 \%$ of patients have venous insufficiency, $15 \%$ have experienced venous ulceration, $15 \%$ have developed venous claudication, $40 \%$ have restricted ambulation and many patients demonstrate hemodynamic impairment and reduced quality of life (QOL) [10, 11].

It is suggested in the American College of Chest Physicians (ACCP) "Antithrombotic Therapy for Venous Thromboembolic Disease" guidelines that patients who develop ilio-femoral DVT and are of low risk of bleeding undergo catheter-directed thrombolytic therapy or operative thrombectomy to prevent iliofemoral chronic obstruction (grade 2B) [12]. However, most patients are treated with anticoagulation alone rather than a strategy of thrombus removal.

Iliac veins are involved in about $40 \%$ of iliofemoral thrombosis [13], while standard treatment fails to achieve sufficient recanalization of these veins in about $70 \%$ of cases [14]. Inadequate spontaneous recanalization of the thrombus causes a persistent outflow obstruction withvenous hypertension and reflux s of femoral and sometimes of deep femoral vein of the affected leg. The resulting post-thrombotic syndrome (PTS) is a chronic debilitating clinical entity characterized by a spectrum of disease severity from chronic leg swelling and pain, to skin changes, claudication, and in severe cases ulceration [14].

Although current management for acute DVT consists of administration of anticoagulation for 3 to 6 months, daily wearing of compressive stockings for two years and immediate mobilisation these interventions do not sufficiently target the existing occlusion. Recent advances in catheter-based interventions have led to the development of a variety of minimally invasive endovascular strategies to remove venous thrombus. Catheter-based endovascular techniques have revolutionized therapeutic options for DVT by altering the risk-benefit ratio of intervention. Various methods now exist including catheter directed thrombolysis (CDT), pharmaco- mechanical CDT (PCDT), and percutaneous mechanical thrombectomy (PMT) $[15,16]$.

In the last decade there has seen an increasing use of aggressive, catheter-based methods for treating deep vein thrombosis (DVT). In this article, we outline the risks, benefits, and uncertainties surrounding endovascular DVT treatment options, describe clinical situations in which endovascular options should reasonably be considered, and show new outcome data that pertains to catheter-based DVT interventions.

Furthermore, based on the results of recent randomized trials this article should provide guidance to practitioners on optimal patients selection and treatment.

\section{Rationale for Catheter Directed Thromboreductive Therapies}

Acute DVT can directly lead to major health consequences in several ways: (1) PE, the presenting manifestation in approximately onethird of venous thromboembolism (VTE) cases, is often fatal; (2) paradoxical embolization, a rare occurrence in which venous thrombus passes through an intracardiac shunt to enter the arterial circulation, can lead to serious systemic embolization including stroke; and (3) phlegmasia cerulea dolens, a rare condition in which DVT causes massive swelling of the entire extremity, can lead to arterial insufficiency, compartment syndrome, venous gangrene, limb amputation with high rate of mortality. In addition, despite the use of anticoagulant therapy annd compressive stockings, late complications include recurrent VTE, chronic thromboembolic pulmonary hypertension, and PTS may occure [17].

PTS develops in 25-50\% of patients with a first episode of proximal lower extremity DVT and typically causes chronic, lifestyle-limiting symptoms such as swelling, pain, heaviness, and fatigue of the affected limb [18]. Patients with severe PTS may experience short-distance venous claudication that precludes significant physical activity, stasis dermatitis, skin changes such as hyperpigmentation and subcutaneous fibrosis, and skin ulceration [19].

Although PTS has been recognized as a clinical entity for years, its prevention has typically been viewed as a low priority treatment objective by physicians managing DVT.

However, it is now known that the presence and severity of PTS are the primary determinant of a DVT patient's late quality of life (QOL), and that the degree of health impairment caused by PTS is comparable to that of chronic diseases such as chronic obstructive pulmonary disease and arthritis [20]. Because established PTS and venous ulcers are difficult and expensive to treat and often result in work disability, the burden of PTS has major economic consequences to society as well [21]. 


\section{Categorizing Patients with Acute Deep Vein Thrombosis}

Treatment for DVT will vary based on clot location and it's extent, thrombosis history, and comorbidities.

Because the risk of developing serious complications such as PE is highest near the time of diagnosis, guidelines recommend the initiation of anticoagulation therapy in patients with high suspicion of DVT even before ultrasound confirmation of diagnosis, in the absence of major risk factors for bleeding [22].

Due to differences in the risk of developing PE and PTS, distal DVT (involving deep veins below the popliteal vein) is managed differently from proximal DVT (involving the popliteal and/or more cephalad deep veins) [23]. As noted in previous research, patients with proximal DVT generally require therapeutic-dose anticoagulation for at least 3 months $[18,19]$. However, less well-appreciated is another anatomic distinction of importance: the subset of proximal DVT patients who have Thrombus involvement of the iliac and/or common femoral vein (CFV) ("iliofemoral DVT") actually have the highest rates of VTE recurrence, PTS, and more severe PTS [19]. Since the vast majority of cases of iliac vein thrombosis extend into the CFV, most of these patients are easily identified on the initial diagnostic ultrasound exam. Hence, it is the authors' view that patients with lower-extremity DVT can and should be grouped into three anatomic categories based on their future risk of PE and PTS: distal DVT, proximal (femoralpopliteal) DVT, or proximal (iliofemoral) DVT.

Clinical severity is also an important consideration. Patients with asymptomatic (incidentally discovered) DVT are at low risk of developing PTS [24]. In contrast, patients with more severe presenting symptoms have greater impairment of early QOL; patients who are highly symptomatic one month after diagnosis are at high risk for PTS [23, 25]. Finally, patients who present with tense limb swelling with cyanosis should undergo urgent vascular evaluation, since patients with phlegmasia cerulea dolens are at high risk for limb amputation.

\section{Stratification of patients' risk of developing PTS}

The predictors of the development of PTS are only partially understood. The occurrence of recurrent ipsilateral DVT is associated with a 2- to -6-fold increased risk of PTS. As one might expect, the quality of anticoagulant therapy delivered probably influences the later development of PTS. In one registry, a 2.5-fold increase in PTS was observed in patients whose international normalized ratio (INR) was non-therapeutic more than $50 \%$ of the time [26]. Although adequate anticoagulation should be viewed as a key PTS prevention measure, it is clear that despite anticoagulation many DVT patients will still develop PTS. Studies have identified relatively minor increase of PTS risk in older patients, increased body mass index, and female gender [27]. The anatomic extent of DVT is an important predictor of a patient's subsequent risk of developing PTS. Many physicians have been aware that PTS develops more frequently in patients with proximal DVT compared with distal DVT. However, many physicians may not be aware of the compelling evidence that now exists to show that all proximal DVT is not equal in terms of the late risk of recurrent VTE and PTS. Of significance, patients with "iliofemoral" DVT, defined as DVT involving the CFV and/or iliac vein (with or without involvement of other veins as well), experience recurrent VTE twice as frequently as patients with less extensive proximal DVT (below the ostium of DFV), and have 2-year PTS rates that exceed $50 \%$ despite the use of anticoagulation and compression [27, 28]. These patients are also more likely to develop severe PTS manifestations such as disabling venous claudication and venous ulcers. Because in most cases of iliofemoral DVT the CFV is involved, these patients are easy to identify from the initial ultrasound that was performed to diagnose the DVT.

\section{The "Open Vein" concept}

The open vein concept is closely connected with an active strategy, the aim of which is to quickly remove the occlusive thrombus (through lysis or extraction). The result of this is a reduction in the incidence rate of recurrence and PTS, by restoring flow and by keeping greater valve integrity. After a DVT attack, "natural" recanalisation occurs by way of spontaneous fibrinolysis; 6 months after a deep vein occlusion has been treated, there is a $60 \%$ chance that the segment will be totally reopened, a $40 \%$ chance that it will be blocked or partially recanalised, and a $50 \%$ chance of valve insufficiency [29]. Six months after a DVT incident, Johnson et al. observe that there is both obstruction and reflux in $65 \%$ of cases. These two conditions occurring together is a serious concern, which multiplies the risk of a PTS by 3.5. The spontaneous recanalisation rate depends on the location of DVT. It is much lower for iliofemoral thromboses than for popliteal or distal thromboses [30]. Arguments that back up the "open vein" concept may now be found in studies on patients with DVT who were treated with anticoagulants alone. Prandoni et al. [31] show in patients who develop PTS after DVT, that the frequency of residual venous thrombosis or popliteal valve reflux is much higher after 6 months (47\% versus $23 \%$ ). Moreover, a randomised study comparing surgical thrombectomy with anticoagulation treatment, confirmed that there was greater venous flow restoration and a reduction in PTS with the "aggressive" option than with anticoagulation treatment alone [32].

\section{Interventional Treatment Options for Deep Vein Thrombosis}

During the $1990 \mathrm{~s}$, technological advances prompted renewed interest in treating DVT using invasive means: (1) improvements in noninvasive vascular imaging, including Duplex ultrasound and helical CT scanning, improved the noninvasive characterization of the extent of VTE; (2) improved ultrasound technology facilitated safe and reliable catheter access into the venous system; and (3) catheter systems were specifically developed for intra-thrombus drug delivery and dispersion [32]. Endovascular device development has continued to evolve quickly, with great focus on marrying catheter-based drug-delivery systems with methods of depo- 
siting mechanical, ultrasound, or thermal energy into the vascular system. Together, these advances have facilitated the removal of acute thrombus from the venous system and pulmonary arteries.

\section{Catheter-directed thrombolysis for DVT}

The advent of image-guided, catheter-directed, intrathrombus drug delivery has been recognized as one potential avenue by which the safety and efficacy of thrombolytic therapy might be enhanced. In DVT patients, the theoretical advantages of using image-guided catheter placement to infuse pharmacologic thrombolytic agents directly into the thrombosed vein are several: (1) the ability to achieve a high intra-thrombus drug concentration and to avoid bypass of the drug around the occluded venous segments via collaterals might help to overcome known challenges in treating occlusive thrombus [33]; (2) the ability to reduce thrombolytic agent dose, treatment time, hospital resource utilization, and bleeding complications by achieving more efficient thrombolysis is attractive (3); and the ability to prevent recurrent DVT episodes by concomitantly using adjunctive catheter-based techniques to treat anatomic venous abnormalities that may have contributed to the initial thrombotic episode may be an additional advantage [34].

Ultrasound (US)-accelerated CDT has been developed to rapidly and completely resolve thrombus, thus decreasing the potential risk for PTS. US waves increase clot permeability by affecting the fibrin strands in thrombosis, thus facilitating the delivery of therapeutic agents into the clot [35, 36]. The Ekosonic Endovascular System (Ekos, Boston Scientific, USA) is an US-accelerated thrombolytic system that combines high-frequency, low-intensity US with simultaneous catheter-directed thrombolytics to accelerate clot dissolution rather than fragmentation in the peripheral vasculature. US-enhanced thrombolytic therapy achieves its effect by first disassociating the fibrin mesh to increase the surface area of the thrombus and the fibers that are available to the thrombolytic agent. Simultaneously, acoustic microstreaming, which is caused by the US waves, drives the thrombolytic agent away from the catheter and deep into the loosened clot. High-frequency, low-intensity US can be used safely in many medical applications but has no lytic effect. However, the combination of directed US with the local administration of thrombolytic agents accelerates the thrombolytic process $[35,36]$.

Currently, the most commonly used fibrinolytic drug for DVT is recombinant tissue plasminogen activator (rt-PA, Genentech, South San Francisco, CA). DVT treatment with reteplase (Centocor, Malvern, PA) and tenecteplase (Genentech, South San Francisco, CA) has also been reported [37-39]. Each of these drugs is FDA approved for other uses and is used off-label for DVT treatment. The drug is infused continuously and directly into the thrombus at a low dose (a typical r t-PA dose is $0.5-1.0 \mathrm{mg} / \mathrm{hr}$ ). During this time, the patient typically receives an intravenous infusion of unfractionated heparin at subtherapeutic levels. Hematocrit, partial thromboplastin time (PTT), platelet count, and in some centers fibrinogen level are obtained every 6-12 hr, and in most centers the patient is closely monitored in an intensive care or stepdown unit. If active bleeding occurs or if labora- tory parameters depart from the expected ranges (e.g. PTT $>100 \mathrm{sec}$, fibrinogen $<100 \mathrm{mg} / \mathrm{dl}$ ), the drug infusion is temporarily or permanently discontinued.

Patients are re-studied venographically at 6-24 hr intervals. If only partial thrombolysis is achieved, an angioplasty balloon is used to grossly macerate the softened residual thrombus to increase its surface area and thereby speed the thrombolytic process. Thrombolytic infusion is then continued. After thrombolysis is completed, venography is repeated and any visualized stenoses are treated with balloon venoplasty and stent placement [34, 35]. Completion multiplane venography or intravascular ultrasound (IVUS) is performed to confirm patency of the venous system. Full-dose anticoagulant therapy is re-started and patients are subsequently transitioned to long-term oral vitamin $\mathrm{K}$ antagonist therapy and are asked to wear compression stockings for PTS prevention.

Four studies have compared the use of CDT + anticoagulant therapy versus anticoagulant therapy alone. Study were published in a multicenter RCT, Catheter-Directed Venous Thrombolysis (CaVenT), outcomes were assessed in 189 patients in Southern Norway with femoral, common femoral, and/or iliac vein DVT who were randomized to receive either standard therapy (anticoagulation and compression) and additional CDT with rt-PA or standard therapy alone [40]. It should be noted that the endovascular protocol featured 1-4 day infusions of $r$ t-PA and did not include the use of percutaneous mechanical thrombectomy devices or frequent use of venous stents, which is different from contemporary practice. But of major significance, the use of additional CDT was associated with a $28 \%$ relative reduction in the risk of PTS over a 2-year follow-up period ( $56 \%$ versus $41 \%, \mathrm{P}<0.047$ ). A total of $3 \%$ of CDT-treated patients experienced major bleeding, with none suffering lasting consequences. As the first published multicenter RCT of any endovascular DVT thrombolysis modality, this study is very significant in providing stronger validation of the open-vein hypothesis and in finding that adjunctive CDT improved long-term patient outcome.

Comerota et al. found that 68 acute ilio-femoral DVT patients who underwent successful CDT had fewer PTS symptoms and superior health-related QOL at mean 16-month follow-up than 30 similar patients who received anticoagulation alone [41]. However, this study was limited by marked age differences in the two cohorts, non-randomized design, and small sample size. In 2001, AbuRahma et al. described a prospective study in which 51 acute iliofemoral DVT patients were permitted to choose to receive adjunctive CDT + anticoagulation or anticoagulation alone. The CDT recipients had more frequent 6 -month venous patency $(83 \%$ versus $24 \%, \mathrm{P}<0.0001)$ and 5 -year symptom resolution (78\% versus $30 \%, P<0.0015$ ) [42]. However, this study was also limited by non-randomized design, performance in a single center, and small sample size. In 2002, Elsharawy described a single-center randomized trial (n: 53) evaluating adjunctive CDT for acute iliofemoral DVT. At 6 months, CDT recipients had a higher rate of normal venous function ( $72 \%$ versus $12 \%, \mathrm{P}<0.001$ ) and less valvular reflux ( $11 \%$ versus $41 \%, \mathrm{P}<0.04)$ [43]. However, this study was limited by single-center performance, small sample size, and reliance upon surrogate indicators of success (physiologic testing) rather than validated measures of clinically important PTS and QOL. 


\section{Pharmacomechanical catheter-directed thrombolysis}

Pharmacomechanical catheter-directed thrombolysis (PCDT) refers to the combination of intrathrombus drug delivery with the use of percutaneous mechanical thrombectomy devices that improve drug dispersion and macerate and/or aspirate thrombus from the venous system.

The use of PCDT is intended to enable faster dispersion of the thrombolytic drug within the thrombus, accelerating successful thrombolysis and thereby improving safety by reducing the needed drug dose and exposure time, which may reduce bleeding complications.

Successful use of PCDT has been described in a number of published observational DVT studies, but no multicenter randomized trials. "First-generation" PCDT techniques, in which thrombectomy devices were used to supplement traditional overnight CDT infusions, resulted in initial treatment safety and efficacy comparable to stand-alone CDT; 40-50\% reductions in drug dose and treatment time; and lower hospital stays, intensive care unit utilizion, and costs [44-46]. In recent years, PCDT methods have been introduced that can enable endovascular DVT therapy to be completed on-table in a single 1-3 hr procedure session without the need for further drug infusions or ICU monitoring. With the "power pulse" technique, the Angiojet Rheolytic Thrombectomy Catheter (Boston Scientific, USA) is first used to forcefully pulse-spray a bolus dose of the thrombolytic drug directly into the thrombus $[16,47]$. The drug is allowed to dwell within the thrombus for 20-30 min, then the Angiojet is used to aspirate the residual thrombus. PCDT with Angiojet catheter is based on the Venturi effect: high-speed saline jets (350-450 km/h) directed in a retrograde fashion indirectly create a negative pressure zone that fragments the thrombus and facilitates aspiration of thrombotic material into the device.

A few published case series suggest that effective initial clot removal can be accomplished in $80-90 \%$ of patients, of whom many may be treated in a single procedure session without ICU utilization. In one small retrospective study of patients receiving PCDT, patients who had $>50 \%$ clot removal with PCDT experienced reduced PTS and better QOL than patients receiving PCDT in whom substantial clot removal was not achieved [48].

Major bleeds are estimated to occur in 2-4\% of patients receiving PCDT. Symptomatic PE has also been observed infrequently but there may be a theoretically increased risk of clot displacement with more mechanically aggressive methods [44-48]. Hence, while there is no clear evidence to support or refute this practice, some physicians utilize a retrievable inferior vena cava (IVC) filter during the peri-procedure period when using singlesession PCDT. If this is done, it is important for the team of physicians (including the person who placed the filter) to ensure filter retrieval as soon as the risk of PE is felt to be diminished.

\section{Percutaneous Mechanical Thrombectomy}

The newest generation of treatments for acute DVT include Percutaneous Mechanical Thrombectomy (PMT) device systems, has been reached consensus regarding their comparative efficacy relative to traditional treatments. Thrombectomy devices vary in the mechanism by which they remove clots, such as the Indigo System's (Penumbra Inc., Alameda, Calif) vacuum aspiration, the Arrow-Tretola (Teleflex Inc., Limeric, Pa) device's rotational mechanism and the Clot Buster Amplatz Thrombectomy Device's (Microvena Corp, White Bear Lake, Minn) combination of rotational and vacuum forces [49]. Endovascular devices such as the Angiovac (Angiodynamics, Queensbury, NY), Aspirex s endovascular system (Straub Medical, Wangs, Switzerland) and Trellis (Medtronic, Minneapolis, Minn) devices have also shown promising results for treating acute and chronic DVT [50-52]. The Clottriever System (Inari Medical Inc., Irvine, Calif) is an endovascular mechanical thrombectomy device designed to remove large clots without the use of thrombolytics in a single session [53]. The Clottriever thrombectomy system is comprised of two components: the Clottriever sheath and the catheter. The sheath has a $13 \mathrm{~F}$ diameter and features a self-expanding nitinol funnel at its tip. The catheter is formed by a nitinol coring element and a braided collection bag for retrieving thrombus. After venography revealed an adequate segment of vein where the funnel tip could expand to its full diameter, the system's $13 \mathrm{~F}$ sheath was introduced with subsequent deployment of its funnel. In cases in which there was no vein segment where the funnel tip could expand to its full diameter, predeployment balloon angioplasty was performed. The system's catheter was then introduced in the sheath and advanced over the wire until the mark located at the level of the nitinol coring element had passed the venous segment containing the more central end of the clot. While maintaining the position of the outer catheter, the coring element and collection bag is deployed. Under direct fluoroscopic visualization, the system is slowly retracted at a rate of 1 to $2 \mathrm{~cm} /$ second until the coring element is aligned inside the funnel tip of the sheath.

Twelve patients were included Benarroch-Gampel J. and colleagues Clottriever study [53]. The cohort was $50 \%$ female with a mean age of 55.6 years. Lytic therapy was not used in any case. Complete clot evacuation was obtained in all patients in a single session without repeat interventions. Symptom resolution before discharge was achieved in $100 \%$ of cases. There was no incidence of postoperative anemia (mean hemoglobin, $10.7 \mathrm{~g} / \mathrm{dL}$ preoperatively vs $10.4 \mathrm{~g} / \mathrm{dL}$ postoperatively) or acute kidney failure (mean creatinine, $0,86 \mathrm{mg} / \mathrm{dL}$ preoperatively vs $0,81 \mathrm{mg} / \mathrm{dL}$ postoperatively). The average length of stay was 2 days (range, 1-4 days) without the need for intensive care admission. At early follow-up, 11 patients $(91.7 \%)$ continued to report significant symptom resolution. Two of 10 patients (20\%) developed recurrent occlusive DVTs on follow-up duplex ultrasound examination.

The Aspirex s endovascular system (Straub Medical, Wangs, Switzerland) is an another device for PMT consists of a rotating over-the-wire device designed for efficient and rapid removal of occluding material. The rotations produce a continuous vacuum inside the catheter, which leads to aspiration of the material into the catheter and transportation into the collecting bag. The Aspirex s device is designed for the use in fresh thrombotic or thromboembolic material since its head itself does not rotate, in the meantime avoiding the risk for vessel trauma, and may therefore being used in acute and subacute DVT. The rotating helix of the 
Aspirex system, which is used for rotational thrombectomy, employs the Archimedes principle. The helix rotates within a thin-walled catheter at a rate of 40000 revolutions per minute, which causes suction. Local thrombi are thus aspirated at the tip of the catheter and removed.

The Jeti Thrombectomy System is an hydrodynamic aspiration device using a front-face catheter to aspirate big volumes of clot. Its flexible catheter design allows to reach small branches as well as bigger lumen like ilio-caval segment.

Due to the internal high pressure saline jet, the Jeti has a minimized risk for hemolysis or embolic complications. Its atraumatic design with the soft tip allow the physician to use it with or without guide wire in place. There are different frech sized available for different indications. Combined with steerable or fixed curved guide sheath the Jeti can reach any spot in venous segment to remove clot from the wall and create bigger lumen, safe and effective [54].

\section{Patient selection for endovascular acute DVT therapy}

A strong preponderance of the available evidence favors the use of CDT or PCDT in DVT patients with (a) clinically severe manifestations of DVT including phlegmasia cerulea dolens, acute IVC thrombosis, or rapid thrombus extension despite anticoagulation or (b) anatomically extensive DVT that includes the common femoral vein and/or iliac vein, since this degree of involvement portends a much higher risk of recurrent DVT and PTS. At present, a highly individualized approach to patient selection is recommended $[15,16,40]$. Major additional factors which impact the decision process include symptom duration; the patient's bleeding risk profile, life-expectancy, and anticipated activity level; and his/her willingness to undergo a minimally invasive catheterbased procedure.

Successful thrombolysis of acute DVT is most likely to be achieved in patients with recently formed thrombus, as evidenced by DVT symptom duration of less than 10-14 days

[35]. The best group of patients is probably those with acute iliofemoral DVT [34-36]; in particular, those with a left-sided descending iliofemoral DVT are likely to have an underlying stenosis ("May-Thurner Syndrome") that can be eliminated with stent placement. Patients with symptom duration 14-28 days usually also experience significant thrombolysis, but such patients often require adjunctive iliac vein stent placement to restore complete venous patency with acceptable flow.

Patients with short life-expectancy, those who do not ambulate, and those with factors predisposing them to bleeding complications (e. g., recent surgery, trauma, or obstetrical delivery; intracranial lesions; severe thrombocytopenia) are poor candidates, as are three additional subsets of patients: (1) patients with chronic DVT (>28 days) limited to the femoropopliteal segment, since thrombolytic therapy tends to be ineffective for them [35]; (2) patients with isolated calf vein DVT, since they tend to be less symptomatic and to develop PTS less frequently [3]; and (3) patients with asymptomatic DVT, since they rarely develop PTS.

\section{Discussion}

The treatment of DVT has advanced significantly in recent years, but major challenges remain. Despite the use of standard anticoagulant therapy, DVT recurs frequently and often leads to the development of permanent sequelae from post-thrombotic syndrome (PTS) [1-3]. Catheter-based techniques have been used in the management of DVT for many years, but only few are undergoing rigorous evaluation in multicenter randomized controlled trials (RCTs) to determine whether they improve patient outcomes.

Standard DVT treatment focuses on adequate anticoagulation to prevent pulmonary embolism and thrombus propagation. However, anticoagulation alone has no direct thrombolytic effect. Current conservative DVT treatments often do not restore venous patency, and venous valves become permanently damaged. The prompt and effective removal of acute thrombosis effectively preserves venous valvular function, reduces reflux, and removes venous obstruction. Additionally, this treatment reduces the risk of thrombus recurrence, which is a strong predictor of a poor long-term outcome of DVT and severe PTS [55].

Multiple approaches have been proposed; however, the focus has shifted towards endovascular interventions for DVT. To date, the results of using endovascular interventions for DVT are encouraging and far exceed those that were obtained using anticoagulation alone in the appropriate patient population. Furthermore, selected patients with established moderate-to-severe PTS in association with an occluded iliac vein or a refluxing femoral vein may also be amenable to endovascular intervention to reduce venous hypertension, alleviate symptoms, and improve limb function and quality of life.

Catheter-directed thrombolysis and PMT are both emerging techniques for treatment of acute DVT of the lower extremities that have the advantage of early restoration of venous flow and thus reduction of post-thrombotic complications. A 2015 metaanalysis compared the efficacy of CDT plus anticoagulation versus that of anticoagulation alone in the treatment of proximal DVT. It showed that additional CDT was associated with significantly improved 6-month venous patency and PTS rates [56].

As compared with regular CDT, PMT is another endovascular option that has provided promising clinical outcomes with better controlled bleeding risk. This review has served as a comprehensive overview of clinical and safety outcomes across different categories of thrombectomy devices. It demonstrated well that the procedural outcomes of both PMT alone and that with pharmacomechanical devices were non-inferior to that of CDT in treatment of acute DVT in the lower extremities. The rates of PTS, bleeding complications, and hospital costs of PMT were all shown to be favorable to those of CDT alone. In addition, the mortality risk of PMT was minimal and comparable to that of patients treated with anticoagulation alone: $0.4 \%$ recurrent fatal venous thromboembolism and $0.2 \%$ fatal major bleeding events [57].

Management of DVT in patients with active cancer remains controversial. It is known that malignancy incurs a state of increased risk of bleeding and recurrence of DVT; one study showed $36 \%$ of active patients with cancer may develop asymptomatic DVT [58]. When managed medically, DVT in patients with 
cancer is best treated with low-molecular-weight heparin over traditional warfarin, but symptomatic presentation may require more urgent intervention. PMT Mdevices, such as the Angiojet or Aspirex, have been shown to be effective in removing acute thrombus in patients with cancer with equivocal results when compared with patients without cancer [59]. Regardless of treatment modality, the decision to treat patients with cancer for DVT must take into account many factors including patient prognosis, appropriateness of therapy and a comparison of the benefits and burdens of treatment. Our experience with this decision-making process was most successful when a multidisciplinary team worked in conjunction with the patient and patient's family.

On the other hand, pregnant women require unique attention in the management of acute thrombotic conditions owing to their hypercoagulable state, increased risk of postpartum hemorrhage, and potential effect of thrombolysis on the fetus [60]. A purely mechanical thrombectomy device or Angiojet PCDT with intravascular Ultrasound (IVUS) support would be preferred for treatment in these groups.

Early thrombus removal techniques include CDT, PCDT and PMT should be used to reduce the risk of PTS. However, early studies of catheter directed techniques have either been retrospective [41], non-randomized [42] or did not evaluate clinically relevant end points [43]. Three modern trials guide contemporary practice. The Catheter-Directed Venous Thrombolysis in Acute Iliofemoral Vein Thrombosis (CaVenT) trial was published in 2012 and the Acute Venous Thrombosis and Thrombus Removal With Adjunctive Catheter-Directed Thrombolysis (ATTRACT) trial was published in 2017 [40, 61]. In CaVenT, CDT reduced the rate of PTS at 2 years from $55.6 \%$ to $41.1 \%$ ). Importantly, the effect became more pronounced at 5 years, with $71 \%$ of the control group and $43 \%$ of the CDT group developing PTS [62]. Severe PTS was present in $1 \%$ of the control group and $5 \%$ of the CDT group, and quality of life scores did not differ between groups. Procedural bleeding complications occurred in 20 patients $(9.6 \%$ risk) without any intracranial hemorrhage. The ATTRACT trial randomized 692 patients with acute femoro-popliteal or iliofemoral DVT to PCDT vs. anticoagulation alone, and did not show a significant difference in the rates of PTS at 2 years ( $47 \%$ vs. $48 \%$ rates of PTS in the PCDT and control groups). There were significantly more bleeding complications in the intervention group (1.7\% vs. $0.3 \%$ in the intervention and control groups) but no intracranial hemorrhages occurred. A subgroup analysis of patients with iliofemoral DVTs also failed to show any significant reduction in the risk of PTS or improvement in quality of life measures with PCDT [63]. However, an analysis of secondary outcomes did show that the proportion of patients with moderate-severe PTS was reduced with PCDT compared to standard treatment (18\% vs. $24 \%(p=0.04))$, and this effect was more pronounced in the iliofemoral subgroup ( $18 \%$ vs. $28 \%(p=0.02)$ ). In the iliofemoral subset, pain and swelling scores at 10 days were significantly lower in the PCDT group (pain score decrease of 1.76 vs. $0.51(p=0.009)$ and leg circumference change of $<0.79$ vs. $0.22(p=0.002)$ in the PCDT and control groups). Echoing the above findings, QOL scores were improved during the first 6 months in patients assigned to the PCDT group [64].
Taken together, the CaVenT, ATTRACT data suggest that catheter directed techniques reduce acute DVT symptoms and may also improve early post-DVT QOL. Based on CaVenT the benefit of CDT might be more apparent in the very long term with a reduction of the risk of PTS.

The management of acute DVT and chronic PTS remains complex, with many possible avenues of improvement. Most importantly, the pathophysiology of PTS remains poorly understood. It is hoped that future studies will include evaluation of genomic factors and other biomarkers to shed light on who is most likely to develop PTS, progress in severity, and benefit from interventions.

An important short-term goal is to continue to educate the medical community on the importance of treatment of acute DVT patients and prevent PTS. It is now many years since PTS was recognized as a major contributor to poor QOL in DVT patients, yet it continues to be neglected as a study outcome of high priority. It is also manifestly clear that patients with iliofemoral DVT constitute a distinctive subgroup that experiences more recurrent VTE, more PTS, and more severe PTS. Increased education is needed to ensure that these patients are identified as "high-risk" at diagnosis, that they receive closer early monitoring to ensure effective anticoagulation and symptom control, and to consider interventional treatment when appropriate. Treatment studies should routinely report outcomes separately in patients with iliofemoral DVT, since they may differ from those of other patients with proximal DVT.

Regarding the use of currently available endovascular treatment options, randomized trials have clarified that selective use, rather than routine use, is most appropriate. Patients with highly symptomatic involvement of the iliofemoral, axillosubclavian, and caval veins should be the focus. Additional analyses would be helpful to determine whether specific subgroups of patients with iliofemoral DVT are most amenable to benefit and could create a case for routine, first-line use. Until then, therapy should be delivered on a highly individualized basis, with a focus on ensuring treatment safety, using best practices to optimize efficacy, and ensuring a patient voice in decision-making.

\section{Conclusion}

Interventional therapies for DVT offer the potential to provide rapid symptom relief and prevention of PTS-related disability. The quality of evidence in support of these treatments continues to improve. In particular, PCDT and PMT treatments show strong potential to provide DVT patients with significant clinical benefits. Randomized trials are underway to determine whether favorable outcomes are indeed obtained using percutaneous methods of treating acute DVT. Until they are completed, a highly individualized approach to patient selection is recommended to ensure patient safety and optimize clinical benefit.

\section{Conflict of Interest}

The authors declare that they have no conflict of interest. 
[1] Vedantham S, Grassi C], Ferral H et al. Reporting Standards for Endovascular Treatment of Lower Extremity Deep Vein Thrombosis. J. Vasc. Interv. Radiol 2006; 17: 417-434

[2] Thomas M, Hollingsworth A, Mofidi R. Endovascular Management of Acute Lower Limb Deep Vein Thrombosis: A Systematic Review and Meta-analysis. Ann. Vasc. Surg 2019; 58: 363-370

[3] Nathan AS, Giri J. Reexamining the Open-Vein Hypothesis for Acute Deep Venous Thrombosis. Circulation 2019; 139: 1174-1176

[4] Tovey C, Wyatt S. Diagnosis, investigation, and management of deep vein thrombosis. BMJ 2003; 326: 1180-1184

[5] Hood DB, Weaver FA, Modrall JG et al. Advances in the treatment of phlegmasia cerulea dolens. Am. J. Surg 1993; 166: 206-210

[6] Ende-Verhaar YM, Tick LW, Klok FA et al. Post-thrombotic syndrome: Short and long-term incidence and risk factors. Thromb. Res 2019; 177: 102-109

[7] Schleimer K, Barbati ME, Grommes J et al. Update on diagnosis and treatment strategies in patients with post-thrombotic syndrome due to chronic venous obstruction and role of endovenous recanalization. J. Vasc. Surg. Venous Lymphat. Disord 2019; 7: 592-600

[8] Kahn SR, Shrier I, Julian JA et al. Determinants and time course of the post- thrombotic syndrome after acute deep venous thrombosis. Ann Intern Med 2008; 149: 698-707

[9] Brandjes DP, Buller HR, Heijboer $\mathrm{H}$ et al. Randomised trial of effect of compression stockings in patients with symptomatic proximal-vein thrombosis. Lancet 1997; 349: 759-762

[10] Prandoni P, Lensing AW, Prins MH et al. Below-knee elastic compression stockings to prevent the post-thrombotic syndrome: A randomized, controlled trial. Ann Intern Med 2004; 141: 249-256

[11] Delis KT, Bountouroglou D, Mansfield AO. Venous claudication in iliofemoral thrombosis: Long-term effects on venous hemodynamics, clinical status, and quality of life. Ann Surg 2004; 239: 118-126

[12] Kahn SR, Ducruet T, Lamping DL et al. Prospective evaluation of healthrelated quality of life in patients with deep venous thrombosis. Arch Intern Med 2005; 165: 1173-1178

[13] Mazzolai L, Aboyans V, Ageno W et al. Diagnosis and management of acute deep vein thrombosis: a joint consensus document from the European society of cardiology working groups of aorta and peripheral circulation and pulmonary circulation and right ventricular function. Eur Heart J 2018; 39 (47): 4208-4218

[14] Vedantham S, Kahn SR, Goldhaber SZ et al. Endovascular therapy for advanced postthrombotic syndrome: Proceedings from a multidisciplinary consensus panel. Vasc Med 2016; 21: 400-407

[15] Fleck D, Albadawi H, Shamoun F et al. Catheter-directed thrombolysis of deep vein thrombosis: literature review and practice considerations. Cardiovasc Diagn Ther 2017; 7 (Suppl. 3): S228-\$237

[16] Dumantepe M, Uyar I. The effect of Angiojet rheolytic thrombectomy in the endovascular treatment of lower extremity deep venous thrombosis. Phlebology 2018; 33 (6): 388-396

[17] Van Dongen CJ], Prandoni P, Frulla M et al. Relation between quality of antcoagulant treatment and the development of the postthrombotic syndrome. J Thromb Haemost 2005; 3: 939-942

[18] Kahn SR. The post-thrombotic syndrome. Thromb Res 2011; 127: (Suppl. 3): S89-S92

[19] Douketis JD, Crowther MA, Foster GA et al. Does the location of thrombosis determine the risk of disease recurrence in patients with proximal deep vein thrombosis? Am J Med 2001; 110: 515-519

[20] Kahn SR, Shbaklo H, Lamping DL et al. Determinants of health-related quality of life during the 2 years following deep vein thrombosis. J Thromb Haemost 2008; 6: 1105-1112

[21] Guanella R, Ducruet T, Johri M et al. Economic burden and cost determinants of deep vein thrombosis during 2 years following diagnosis: A prospective evaluation. J Thromb Haemost 2011; 9: 2397-2405

[22] Kearon C, Akl EA, Comerota AJ et al. Antithrombotic Therapy for VTE Disease: Antithrombotic Therapy and Prevention of Thrombosis, 9th ed: American College of Chest Physicians Evidence-Based Clinical Practice Guidelines. Chest 2012; 141: 419-496

[23] Endig H, Michalski F, Beyer-Westendorf ]. Deep Vein Thrombosis-Current Management Strategies. Clin. Med. Insights Ther 2016; 8: doi:10.4137/CMT.S18890

[24] Vedantham S, Goldhaber SZ, Julian JA et al. Pharmacomechanical Catheter-Directed Thrombolysis for Deep-Vein Thrombosis. N. Engl. J. Med 2017; 377: 2240-2252

[25] Robert-Ebadi H, Righini M. Should we diagnose and treat distal deep vein thrombosis? Hematol. Am. Soc. Hematol. Educ. Program 2017; 2017: 231-236

[26] Kearon C, Kahn SR, Agnelli G et al. Antithrombotic therapy for Venous thromboembolic disease: American College of Chest Physicians Evidence-Based Clinical Practice Guidelines (8th Edition). Chest 2008; 133: 454S-545S

[27] Vedantham S, Thorpe PE, Cardella JF et al. Quality improvement guidelines for the treatment of lower extremity deep vein thrombosis with use of endovascular thrombus removal. J Vasc Interv Radiol 2006; 17: 435-448

[28] Jaff MR, McMurtry MS, Archer SL et al. Management of massive and submassive pulmonary embolism, iliofemoral deep vein thrombosis, and Chronic thromboembolic pulmonary hypertension: A scientific statement from the American Heart Association. Circulation 2011; 123 : 1788-1830

[29] Roumen-Klappe EM, Janssen MC, van Rossum J et al. Inflammation in deep vein thrombosis and the development of the post-thrombotic syndrome: a prospective study. J Thromb Haemost 2009; 7: 582-587

[30] Johnson BF, Manzo RA, Bergelin RO et al. Relationship between changes in the deep venous system and the development of the post-thrombotic syndrome after an acute episode of lower limb deep vein thrombosis: a one to six years followup. J Vasc Surg 1995; 2: 307-312

[31] Prandoni P, Lensing AW, Prins MH et al. Below-knee elastic compression stockings to prevent the post-thrombotic syndrome: a randomized, controlled study. Ann Intern Med 2004; 141: 249-256

[32] Plate G, Eklöf B, Norgren L et al. Venous thrombectomy for iliofemoral vein thrombosis: 10 -year results of a prospective randomised study. Eur J Vasc Endovasc Surg 1997; 14: 367-374

[33] Meyerovitz MF, Polak FJ, Goldhaber SZ. Short-term response to thrombolytic therapy in deep venous thrombosis: Predictive value of venographic appearance. Radiology 1992; 184: 345-348

[34] Vedantham S, Grassi C], Ferral H et al. Reporting standards for endovascular treatment of lower extremity deep vein thrombosis. J Vasc Interv Radiol 2006; 17: 417-434

[35] Dumantepe M, Tarhan A, Yurdakul I et al. US-accelerated catheterdirected ombolysis for the treatment of deep venous thrombosis. Diagn Interv Radiol 2013; 19 (3): 251-258

[36] Parikh SR, Raabe RA, Motarjeme A et al. Ultrasound-accelerated thrombolysis (UAT) for deep venous thrombosis (DVT): EKOS multicenter experience. J Vasc Interv Radiol Suppl 2006; 17 (2): S49

[37] Sugimoto K, Hoffman LV, Razavi MK et al. The safety, efficacy, and Pharmaco-economics of low-dose alteplase compared with urokinase for catheter-directed thrombolysis of arterial and venous occlusions. J Vasc Surg 2003; 37: 512-517

[38] Castaneda F, Li R, Young K et al. Catheter-directed thrombolysis in deep venous thrombosis with use of reteplase: Immediate results and complications from a pilot study. J Vasc Interv Radiol 2002; 13: 577-580

[39] Razavi MK, Wong H, Kee ST et al. Initial clinical results of tenecteplase (TNK) in catheter-directed thrombolytic therapy. J Endovasc Ther 2002; 9: 593-598 
[40] Enden T, Haig Y, Klow N et al. Long-term outcomes after additional catheter-directed thrombolysis versus standard treatment for acute iliofemoral deep vein thrombosis (the CaVenT study): A randomised controlled trial. Lancet 2012; 379: 31-38

[41] Comerota AJ, Throm RC, Mathias SD et al. Catheter-directed thrombolysis for iliofemoral deep venous thrombosis improves health-related quality of life. J Vasc Surg 2000; 32: 130-137

[42] AbuRahma AF, Perkins SE, Wulu JT et al. Iliofemoral deep vein thrombosis: Conventional therapy versus lysis and percutaneous transluminal angioplasty and stenting. Ann Surg 2001; 233: 752-760

[43] Elsharawy M, Elzayat E. Early results of thrombolysis vs anticoagulation in iliofemoral venous thrombosis. A randomised clinical trial. Eur J Vasc Endovasc Surg 2002; 24: 209-214

[44] Vedantham S, Vesely TM, Sicard GA et al. Pharmacomechanical thrombolysis and early stent placement for iliofemoral deep vein thrombosis. J Vasc Interv Radiol 2004; 15: 565-574

[45] Kim HS, Patra A, Paxton BE et al. Adjunctive percutaneous mechanical thrombectomy for lower-extremity deep vein thrombosis: Clinical and economic outcomes. J Vasc Interv Radiol 2006; 17: 1099-1104

[46] Bush RL, Lin PH, Bates JT et al. Pharmacomechanical thrombectomy for treatment of symptomatic lower extremity deep venous thrombosis: Safety and feasibility study. J Vasc Surg 2004; 40: 965-970

[47] Cynamon J, Stein EG, Dym J et al. A new method for aggressive management of deep vein thrombosis: Retrospective study of the power pulse technique. J Vasc Interv Radiol 2006; 17: 1043-1049

[48] Grewal NK, Martinez JT, Andrews L et al. Quantity of clot lysed after heterdirected thrombolysis for iliofemoral deep venous thrombosis correlates with postthrombotic morbidity. J Vasc Surg 2010; 51: 1209-1214

[49] Delomez M, Beregi JP, Willoteaux S et al. Mechanical thrombectomy in patients with deep venous thrombosis. Cardiovasc Intervent Radiol 2001; 24: 42-48

[50] Jabaar AA, Jenkins JS. The role of vacuum assisted thrombectomy (AngioVac) in treating chronic venous thromboembolic disease. Systematic review and a single center's experience. Cardiovasc Revasc Med 2018; 19: 799-804

[51] Lichtenberg M, Stahlhoff WF, Özkapi A et al. Safety, procedural success and outcome of the Aspirex S endovascular thrombectomy system in the treatment of iliofemoral deep vein thrombosis- data from the Arnsberg Aspirex registry. Vasa 2019 Jul 48 (4): 341-346

[52] O'Sullivan G], Lohan DG, Gough N et al. Pharmacomechanical thrombectomy of acute deep vein thrombosis with the Trellis- 8 isolated thrombolysis catheter. J Vasc Interv Radiol 2007; 18: 715-724
[53] Benarroch-Gampel ], Pujari A, Aizpuru M et al. Technical success and short-term outcomes after treatment of lower extremity deep vein thrombosis with the ClotTriever system: A preliminary experience. J Vasc Surg Venous Lymphat Disord 2020; 8 (2): 174-181

[54] Cournoyer-Rodrigue J, Bui TB, Gilbert P et al. Percutaneous Thrombectomy with the JETi8 Peripheral Thrombectomy System for the Treatment of Deep Vein Thrombosis. J Vasc Interv Radiol 2020; 31 (3): 444-453

[55] Hull RD, Marder V], Mah AF et al. Quantitative assessment of thrombus burden predicts the outcome of treatment for venous thrombosis: A systematic review. Am J Med 2005; 118: 456-464

[56] Du GC, Zhang MC, Zhao JC. Catheter-directed thrombolysis plus anticoagulation versus anticoagulation alone in the treatment of proximal deep vein thrombosis-a meta-analysis. Vasa 2015; 44: 195-202

[57] Carrier M, Le Gal G, Wells PS et al. Systematic review: case-fatality rates of recurrent venous thromboembolism and major bleeding events among patients treated for venous thromboembolism. Ann Intern Med 2010; 152: 578-589

[58] White C, Noble SIR, Watson M et al. Prevalence, symptom burden, and natural history of deep vein thrombosis in people with advanced cancer in specialist palliative care units (HIDDen): a prospective longitudinal observational study. Lancet Haematol 2019; 6: e79-88

[59] Yoon W], Halandras P, Aulivola B et al. Malignancy does not affect outcomes of pharmacomechanical thrombolysis in acute symptomatic iliofemoral deep vein thrombosis. Ann Vasc Surg 2018; 51: 234-238

[60] Fogerty AE. Management of venous thromboembolism in pregnancy. Curr Treat Options Cardiovasc Med 2018; 20: 69

[61] Vedantham S, Goldhaber SZ, Julian JA et al. Pharmacomechanical catheter-directed thrombolysis for deep-vein thrombosis. N. Engl. J. Med 2017; 377: 2240-2252

[62] Haig Y, Enden T, Grøtta O et al. Post-thrombotic syndrome after catheter-directed thrombolysis for deep vein thrombosis (CaVenT): 5-year follow-up results of an open-label, randomised controlled trial. Lancet Haematol 2016; 3: 64-71

[63] Comerota AJ, Kearon C, Gu CS et al. Endovascular Thrombus Removal for Acute Iliofemoral Deep Vein Thrombosis: Analysis from a Stratified Multicenter Randomized Trial. Circulation 2019; 139: 1162-1173

[64] Kahn SR, Julian JA, Kearon C et al. Quality of life after pharmacomechanical catheter-directed thrombolysis for proximal deep venous thrombosis. J. Vasc. Surg. Venous Lymphat. Disord 2020; 8: 8-23 